\title{
Automated Packing Systems-A Systems Engineering Approach
}

\author{
Paul F. Whelan, Member, IEEE, and Bruce G. Batchelor
}

\begin{abstract}
The ability to manipulate previously unseen objects under visual control is one of the key tasks in the successful implementation of robotic, automated assembly and adaptive material handling systems. The automation of such complex industrial environments will require the development of machine vision systems that are highly adaptable and capable of dealing with a wide range of variable products. An important group of applications of this type is found in the automated packing and nesting of arbitrary shapes. The aim of this work has been to produce an efficient packing strategy that is flexible enough for a wide variety of industrial uses and which can be implemented using fast moderately priced hardware. A systems approach, as distinct from a purely algorithmic one, has been deliberately adopted since the work is concerned with industrial vision applications in which significant problem constraints exist. This paper also outlines the background to this research, and reviews a selection of industrial packing applications.

The packing procedure that has been devised, consists of two major components. The first is a geometric packing technique that is based on morphological image processing operations. This is used in conjunction with a Prolog based heuristic packing procedure. Some of the factors considered at the heuristic level include shape ordering and shape orientation, both of which must be carried out prior to the implementation of the geometric packer. The heuristic procedures deal with problem constraints that are specific to a given application.
\end{abstract}

\section{INTRODUCTION}

$\mathbf{T}$ HE development of flexible vision systems will play a key role in the progression of industrial automation. To achieve a high degree of self-reliance in automatic assembly machines, it will be necessary to find engineering solutions to a wide range of long-standing industrial parts-handling problems. Key considerations include the ability for such systems to manipulate arbitrary, previously unseen objects and to cope with unanticipated situations. Despite the fact that present-day industrial vision systems have some serious limitations [1] vision will, no doubt, have a key role in reaching this end.

\section{A. The General Packing Problem}

The question of automated packing and nesting is not only of theoretical importance, but one with a considerable

Manuscript received June 6, 1994; revised April 26, 1995, and September 3, 1995. This work was supported in part by Eolas and the British Council, C Project 9351.

P. F. Whelan is with the Vision Systems Group, School of Electronic Engineering, Dublin City University, Dublin 9, Ireland (e-mail: whelanp@eeng.dcu.ie).

B. G. Batchelor is with the Department of Computing Mathematics, Machine Vision and Geometric Computing Group, University of Wales, College of Cardiff. Wales, U.K. (e-mail: bruce@ cm.cf.ac.uk).

Publisher Item Identifier S 1083-4427(96)04760-1. industrial interest. Initial motivation comes from viewing specific packing problems:

- How to maximize the number of nonuniform boxes on a pallet?

- How to control a robot packing objects into a box, when no prior knowledge of the shape or size of the objects is assumed?

- How to minimize the number of shipping containers used to transport a given set of items [2]?

A rich theoretical background to such packing problems can be found in scientific, operations research and engineering literature, dating back over 30 years. The theoretical problems associated with automated packing are also complex:

- Does an optimal packing configuration exist for a given application?

- How can shapes be represented for efficient packing?

- What is meant by an efficient packing system and how is the performance of such a system measured?

- How does an automated packing system deal with the arrival of shapes in a random orientation?

- What order should the shapes be presented to the system to ensure an efficient packing strategy?

In today's competitive market, product packaging is playing a more important role than ever before. Changing packet designs, shorter times to the market place, and frequent product introductions are causing manufacturers world wide to change their approach to the packing and packaging process [3]. In the past, manufacturers have relied on traditional packaging technologies, such as dedicated machinery and manual production techniques. Unfortunately, dedicated equipment cannot always meet today's needs for increased production flexibility, and with higher labor and liability costs, manual alternatives are not always a competitive solution. This calls for a new approach to the packing and packaging problem, one which uses automation, but which also provides the flexibility of a manual operator. A key step in developing such a flexible packing system is the integration of intelligent vision feedback.

When designing such a system it is of paramount importance to choose a strategy that will deal with the practical constraints involved in automated packing, even if it means sacrificing some volumetric efficiency. This highlights the importance of developing an adaptive packing system within a systems engineering context (that is an environment that considers the application domain). By adopting a systems approach, maximum use is made of problem-specific contextual information derived, for example, from the nature of the product being handled, the manufacturing process and the special features 
of the manufacturing environment. By doing so, we hope to reduce the complexity of the application. For example, in certain packing applications the items must be packed as they arrive at the packing station, therefore, removing the object ordering burden from the packing system. In fact, by taking heed of such constraints in a practical packing application, the packing strategy might well reduce to a standard, welltried technique. It is our belief that in packing, as happens so often elsewhere, systems considerations are always worth investigating.

\section{B. Overview of the Paper}

This paper is concerned with the issues involved in the automated packing of two dimensional objects into a two dimensional bounded region, where the size and shape of both the objects and the region in which they are to be packed are not known prior to packing. The paper has two distinguishable, but interwoven themes: i) maximizing the use of the context information available from a given task, by the application of a systems engineering approach to the problem, and ii) the development of an adaptive packing strategy for random shapes, using morphological and heuristic techniques. Although the systems issues are discussed throughout the paper, Sections III and VI place particular emphasis on these concerns. The latter theme is primarily discussed in Section IV.

Research has shown that an optimal algorithmic solution for even the simplest, well defined packing problem, such as pallet packing, is unlikely to exist [2]. However, the aim of the system described in this work is more ambitious and complex. It's objective is to allow the flexible packing of random two dimensional shapes into previously undefined scenes (the term scene is used when referring to a region of space into which we are required to place an arbitrary shape). The NP-complete nature of the simpler packing problem has a major bearing on the line of research taken. Any attempt at developing an optimal solution to the packing of random shapes, even if it did exist, would be difficult. It would also be difficult to constrain the problem, especially considering that it must deal with unpredictable shapes. Hence our aim has been to produce an efficient packing strategy that is flexible enough for industrial use. To achieve this objective the systems approach to the packing problem is essential.

Other approaches to the packing problem include, single pattern techniques such as dynamic programming, and multipattern strategies such as linear programming [4], [5]. Unfortunately these techniques do not meet all the requirements of a flexible packing system. In the former case, the aim is the generation of an optimal solution, and as such the approach would not seem to hold promise. The second technique, while useful for one dimensional packing applications, is difficult to implement and use in two dimensions. Alternatively, a heuristic approach to packing can be adopted. This line of research would seem to be the way forward if the system is to remain flexible and have the ability to cope with random shapes.

\section{An Overview of the Packing System}

The packing scheme consists of two major components. The first is referred to as the geometric packer, and is based upon the principles of mathematical morphology. This component takes an arbitrary shape in a given orientation and puts the shape into place, in that orientation. A key element in the success of this approach, is that it removes the limitations imposed by having to recognize and describe the object under analysis in order to pack it, thus increasing the systems flexibility.

The second component is referred to as the heuristic packer, and is concerned with the ordering and alignment of shapes prior to the application of the geometric packer. This component also deals with other general considerations, such as the conflicts in problem constraints and the measurement of packing performance. In addition, it deals with practical considerations, such as the effects of the robot gripper configuration on the packing strategy, packing in the presence of defective regions, anisotropy (grain in the material being handled) and pattern matching considerations.

By using heuristics in the packing strategy it is hoped to produce an efficient, but not necessarily optimal solution. However, the main problem with such an approach is that there is a tendency to generate a set of overly complex rules, incorporating a variety of paradoxes and logical conflicts. It is necessary, therefore, to keep all the logic decisions as simple as possible. Another key aspect of applying heuristics to any complex problem, is knowing when the solution is good enough so that the process can be terminated and a result produced [6]. To this end, a mechanism for the measurement of the packing systems performance must also be included in the overall system design.

\section{Automated Packing Strategies}

This section outlines the current research into the development of techniques and systems for the automated packing of both regular and irregular shapes in industrial applications. It is not the aim of this review to try and cover all the various elements discussed in the literature relevant to packing and nesting, as this has been done extensively elsewhere [2], [5], [7]. The aim of this review is to concentrate on the elements in the literature that are relevant to industrial packing.

\section{A. Review of Current Research}

The survey by Dowsland and Dowsland [5] is one of the more complete, and recent reviews of the application of operational research techniques to the solution of two and three-dimensional packing problems such as pallet packing and container loading. As well as modeling and solving problems, the authors review a number of algorithmic and heuristic approaches. The emphasis of this work is practical solutions to real issues. Sweeney and Paternoster [4] produced a recent review of the stock cutting and packing problem. This review contains over 400 categorized application oriented references including books, dissertations and working papers. The authors have also grouped the publications according to the three main solution methodologies, these are summarized below: 


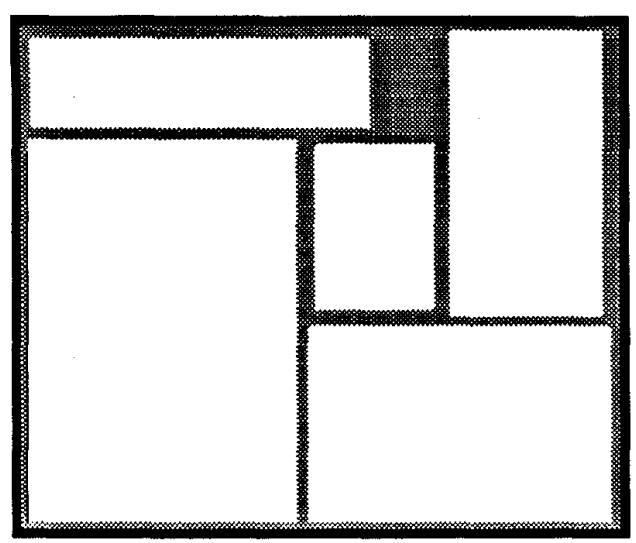

(a)

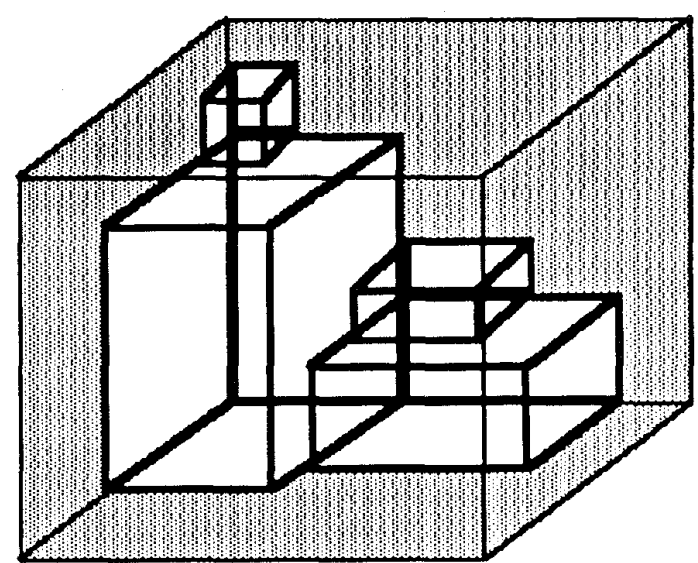

(b)

Fig. 1. (a) Distributors pallet packing. Cartons of varying dimensions are packed on a standard pallet so as to minimize the unused pallet space. (b) Container packing.

- Sequential assignment heuristics, packing of patterns based on a set of assignment rules. The majority of heuristic approaches consist of determining what order and orientation the pieces should be packed in.

- Single-pattern generating procedures such as dynamic programming based algorithms which try and reuse a single optimal packing configuration. For example in the two dimensional rectangular packing problem, the solution is built up by considering partial solutions within smaller containing rectangles [5].

- Multi-pattern generating procedures such as linear programming based approaches which consider the interaction between patterns. This approach requires the solutions to be rounded and are, therefore, also heuristic in nature [8]. The packing task can also be formulated as a binary integer programming problem in which a single variable represents each possible shape position. A major concern with this approach is the production of a physical design from the values of the variables in the integer programming solution [5].

Dychoff [7] develops a consistent and systematic approach for integrating various kinds of cutting and packing tasks to try and unify the various concepts found in the operational research literature. By doing so the author attempts to find the appropriate methods for each relevant problem type and conversely to identify problem types that can be solved by a certain method. A practical review of two and threedimensional packing issues and solution methods can be found in Dowsland's [2] paper. The majority of the applications outlined in this review are based on two dimensional packing techniques. Many of the three-dimensional problems are tackled by applying two dimensional techniques on a layer by layer basis. Most published work in the area of threedimensional packing is limited due to its complexity, and the applications that are discussed tend to be concerned with the loading of shipping containers. The paper also summarizes some of the practical requirements in pallet loading, these include the stability of the loading stack, the load bearing ability of the items in the stack, ease of stacking and the air circulation requirements of certain products in a stack.

Dowsland [2] reviews some of the heuristic approaches used for packing a given set of identical rectangular items into a containing rectangle. A summary of the techniques used in the packing of nonidentical rectangles is also included. This extensive review covers the key areas in automated packing, such as optimality versus efficiency and the measurement of a packing systems performance. The basic conclusion of the author is that although some very high packing densities have been reported in the literature, as yet there is no generic heuristic approach that can be applied to the two dimensional packing task. Solutions reported tend to be very application specific.

\section{B. Packing of Regular Shapes}

The main emphasis of the early research into packing issues tended to concentrate on the well constrained problem of packing regular shapes. This task usually consists of packing two dimensional regular shapes into a well defined scene, such as a rectangle [8]-[14]. The main industrial applications are in the area of pallet packing [11], [12], and container loading [15], see Fig. 1. Other applications include efficient VLSI design and automated warehousing [16], [17].

Hall, Shell, and Slutzky's [16] work combines automated packing techniques developed in the field of operational research, with systems engineering and artificial intelligence approaches to packing. It outlines the issues associated with the arrival of packages at the packing station and relates this to the single server queuing problem which is commonly discussed in the operational research literature. The authors also discuss a number of systems issues, such as the importance of the product information. A practical example would be the packing of food stuffs and toxic products. In this case the packing strategy has not only to consider the efficiency of the packing procedure, it must also consider the product type. The food stuffs and the toxic products should be packed in different boxes. These boxes should be well separated on the pallet to prevent contamination of the food. The authors also highlight 
the importance of how the pallet data is represented, and how to determine the correct placement location for the robot. Other related areas of research discussed include, bin picking, automated storage and retrieval, automated kitting of parts for assembly, automated warehousing, and line balancing [7].

Bischoff [18] discusses the methodologies of the pallet and container packing problem. The main emphasis of this paper is a discussion of the techniques used in the interactive tuning of packing algorithms. The author points out that pallet packing stability criterion is application dependent. If the pallet load is wrapped or strapped down, then this criteria is less important. This is a significant point as there is often a conflict between stability constraint and need to minimize waste space on the pallet. The concept of "cargo fragility" in container loading, and its relationship to the stability requirements, is also discussed.

\section{Packing of Irregular Shapes}

More recently researchers have begun to concentrate on the issues involved in the packing of irregular shapes. Batchelor [19] outlines a technique for the packing of irregular shapes based on the use of the minimum area bounding rectangle In this approach, each shape is enclosed by its minimum fit bounding rectangle, and these rectangles are packed using the techniques developed for the packing of regular shapes. Qu and Sanders [20] discuss a heuristic nesting algorithm for irregular parts and the factors affecting trim loss. The application discussed is the cutting of a bill-of-materials from rectangular stock sheets. The authors take a systems approach to the problem and produce some good results. These are discussed in the context of performance measurements which they have developed. While the authors review the published work in this area, they make the important point that although a number of techniques have been developed to enable the flexible packing of irregular shapes, very few of these have been published due to commercial confidentiality.

Qu and Sanders [20] describe irregular shapes in terms of a set of nonoverlapping rectangles. The authors state that each of the parts in their study can be represented by no more than five nonoverlapping orthogonal rectangles. The system places each part in an orientation such that, a) its length $>$ height, and b) the largest complimentary (void) area is in the upper right corner. The parts are then sorted by nonincreasing part height. The shapes are packed into a rectangular scene in a raster fashion, building up layers of intermeshed packed shapes. The major disadvantage with this approach are: a) the use of rectangles to approximate the shape to be packed, and b) the assumption that good packing patterns will be orthogonal.

Dori and Ben-Bassat [21] and Chazelle [22] were the first to investigate the nesting of shapes within a polygon rather than a rectangle. The authors discuss the optimal packing of two dimensional polygons with a view to minimizing waste. The algorithm is only applicable to the nesting of congruent convex figures. The problem involves cutting a number of similar but irregular pieces from a steel board, this is referred to as the template-layout problem. The authors decompose the task into two subproblems. The first consists of the optimal (minimal waste) circumscription of the original irregular shape by the most appropriate convex polygon. The remaining problem consists of circumscribing the convex polygon by another polygon that can pave the plane, that is, cover the plane by replications of the same figure without gaps or overlap. This is referred to as the paver polygon. Limitations of this approach include the fact that it is only applicable to congruent convex figures and the assumption that the packing plane is infinite, hence, waste in the margin is not considered. Another limitation of this approach is that it can only be applied to convex components with straight sides.

Koroupi and Loftus [23] address the issues raised by Dori and Ben-Bassat [21], by enclosing the component within a polygon so that the area added is minimal. The identical components, whether regular or irregular, are then nested using paving techniques. Martin and Stephenson's [24] paper deals with the packing of two and three-dimensional objects into a well defined scene. In this paper, the authors tackle the task of automated nesting from a computer-aided design perspective. That is, given an arbitrary polygon and a rectangular box, will the polygon fit in the box, and if so how should the polygon be translated and rotated to implement this fitting.

Prasad and Somasundaram [25] outline a heuristic based computer aided system that will allow the nesting of irregular shaped sheet metal blanks. This paper also contains a comprehensive list of the practical constraints one must consider in developing a packing system for sheet metal stamping operations. Constraints such as bridge width, blank separation, grain orientation, and the minimization of scrap. They also highlight the need to align the pressure center of the blank to be cut out with the axis of the press ram to reduce wear in the guideways of the press. Design requirements, such as maximizing the strength of the part when subsequent bending is involved, are also considered.

Chow [26] discusses the optimal packing of templates of uniform orientation under limited conditions. This paper is useful as it discusses the edge effect issues in packing, which in general tend to be neglected. The author also outlines some of the concerns associated with manual packing. Kothari and Klinkhachorn [27] present a two dimensional packing strategy capable of achieving dense packing of convex polygon shapes. The techniques described have been applied to the stock cutting in the hardwood manufacturing industry. This consists of efficiently cutting wooden pieces from a hardwood board so that the pieces are free of defects and aligned in the direction of the grain. This last constraint is needed for strength and aesthetic reasons.

Albano and Sapuppo [28] outline a procedure which will produce an optimal arrangement of irregular pieces. Manual and semiautomatic approaches to this nesting task are also discussed. The techniques described show how the optimal allocation of a set of irregular pieces can be transformed into the problem of finding an optimal path through a space of problem states from the initial state to the goal state. The search approach developed makes certain assumptions about the task; a) the pieces are irregular polygons without holes, and b) that the scene is rectangular. The main applications discussed is that of cloth layout and leather cutting. 


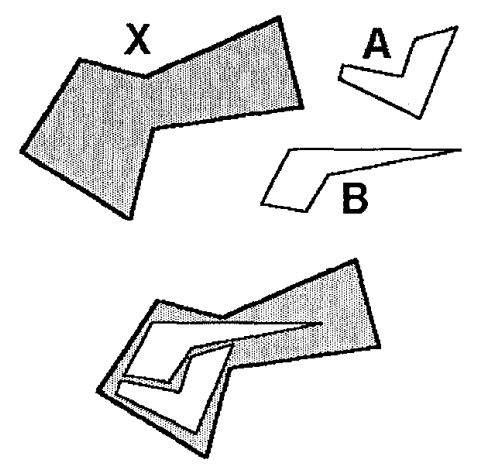

Fig. 2. Tailor suit problem [29].

Vincent [29] discusses the application of morphological techniques to the tailor suit or space allocation problem, see Fig. 2. This addresses the problem of translating two shape pieces, $A$ and $B$, such that both are included in a larger shape piece $X$ without overlapping. Although this can be shown mathematically for two pieces [29], there is no general solution to this problem involving simple morphological techniques only. While the author does not make the link between this technique and the automated packing and nesting in an industrial system, the use of such a powerful technique to manipulate shapes does seem to point the way forward.

\section{The Role of Automated PACKING IN INDUSTRIAL SYSTEMS}

Automated packing/nesting and automated assembly are closely related tasks. In the majority of assembly systems, the parts only fit together in certain ways, dependant on their shapes, and they can only be moved into their fitting positions in ways that are also dependent on their shape. This shape dependent part fitting is a key feature in any assembly application [30]. A primary source of difficulty in automated assembly is the uncertainty in the relative position of the parts being assembled. This uncertainty can be significantly reduced by the use of vision and tactile sensors. A number of interesting techniques have been developed by engineers tackling automated assembly tasks in puzzle solving [31]-[34] and industrial environments [11].

Lately a growing number of researchers have begun to develop industrial systems that deal with complex robotic tasks [35]. One such application of this new generation of intelligent robotics is the automated packing (nesting) of parts in an assembly process. Applications of these systems include the automated assembly of small components under visual control [36], air motor assembly [3], automated gasket and carburetor mating [37], and automatic shirt collar assembly [38].

Hall, Slutzky, and Shell [39] give a good overview of the application of part nesting in intelligent robotic packaging and processing systems, as well as outlining robotic game playing systems and actual solutions to a number of industrial problems. A system for the automated palletising of randomly arriving parcels, has been developed by the authors. The authors have taken account of some of the various systems issues involved in the design of industrial packing systems. They discuss the heuristics necessary to deal with the in- terlocking and intermeshing of the boxes on the pallets as well as dealing with the toxicity and crushability of the boxes contents. Other areas of related research include the work of Jain and Donath [40], in which they discuss the development and implementation of a knowledge based system for threedimensional automated assembly tasks under robotic control.

Hoffman [41] examines eliminating the requirement to hand tune specific assembly tasks in an automation environment. Hitakawa [42] outlines the development of a SONY flexible automatic assembly system suitable for small quantity batch production. Philip Chen [43] tackles the problem of trying to find all the feasible assembly sequences for a set of $n$ parts that construct a mechanical object. Kak et. al. [44] outline the development of a knowledge based robotic assembly cell. This cell is used in the sensory guided part mating of three-dimensional objects. Ayache and Faugeras's [45] paper discusses the development of a robotic system under machine vision control. This system carries out the automated picking and placement of partially overlapping industrial parts. The system is based on the generation and recursive evaluation of hypotheses for object recognition.

Some of the practical considerations found in the industrial implementation of automated packing systems are summarized below:

- Packing density/stability.

- Object fragility/rigidity/clampabilty.

- Surface finish (objects with a smooth finish will be easier to slide/push).

- Ease of stacking.

- Load bearing ability, and the effect of dynamic forces.

- Interaction between the packaging material and its contents.

- Electromagnetic radiation.

- Possibility of contamination of adjacent goods.

- Importance/value of the article.

- Protection from pilferaging.

- Need to provide ullage or vacuity.

- Probable number, and nature of loadings and unloadings.

- Minimization of robot arm movement during loading and unloading.

- Material handling facilities at the final destination.

- Climatic considerations.

- Air circulation.

- Packing for aesthetic appeal (do all products need to be visible?).

- Design and functionality of the handling equipment (e.g., robot gripper).

Tables I-IV summarize the potential and actual use of vision based automated packing and assembly systems in a variety of industries.

The main conclusion that can be drawn from current research is that an optimal algorithmic solution for even the simplest, well defined packing problem, such as pallet packing, is unlikely. This has a major bearing on the research direction taken. Any attempt at developing an optimal solution, even if it did exist, would be difficult, due to the NP-complete nature of the problem. The development of a partial algorithmic solution 
TABLE I

Automated AsSEMBLy APPLICATIONS

- Visual control in robotic assembly:

- Small part assembly [36].

- Automatic bolt insertion [46].

- 'Peg in the hole' [47].

- Fuel pump [48].

- Air motor assembly [3].

- Gasket and carburettor mating [37].

- Push buttons [49].

- Car body assembly [50].

- 3D part mating [44].

- Shirt collar assembly [38].

- Automated component placement:

- Electronic components on a PCB [48].

- Insertion of DIL packages [51].

- Capacitor assembly [3].

- Packing for ease of assembly.

- Piece placement prior to welding under visual control

- Parts kitting [52].

- Assembly of deformable objects [53].

- Fixtureless assembly/manufacture [54].

- Puzzle assembly:

- Jigsaws [31], [32], [33].

- Chinese tangrams [34].

- 3D Soma puzzle [30].

- Peg solitaire game [39].

- Hazardous assembly tasks.

TABLE II

PaCKING AND UnPaCking APPlications

- Packing very variable products:

- Fruit and vegetables.

Soft fruit [55], Stacking oranges [56].

- Food and agricultural products.

Chicken pieces [57], Prawns [58].

- Horticulture.

Packing plantlets and seedlings in a nursery [59].

- Packing in preformed trays [3], [48], [60].

- Retail packaging.

- Packing / packaging for aesthetic appeal

- Packaging dunnage [54].

- Efficient packing / storage of waste materials.

- Packing dangerous materials.

to the packing task would weaken the flexibility, and hence the usefulness, of such an implementation. It would also be difficult to constrain the problem considering that it must deal with unpredictable shapes.

Another key element in the design of the system concerns the method of shape description. A review of the current literature indicates that the majority of existing systems rely on correlation methods and low level features such as curve and/or critical point matching techniques. Other techniques enclose the shape of interest within a bounding rectangle, polygon or convex hull, prior to paving the region to be packed with these predefined regions. One technique that shows promise is based on the application of mathematical
TABLE III

Storage and Transportation Applications

- Automated warehousing [16], [17].

- Storage retrieval.

- Bin packing [10].

- Container packing for lorries and ships [15], [61].

- Kit packing [17].

- Pallet packing [11], [12].

- Packing to avoid movement during transit.

TABLE IV

Matertal Cutting Applications

- Stock (Bill-of-Materials) cutting [20]:

- Wood [27], Steel [25], Glass [62], Metal rods [19].

- Paper and pulp [8], Piping [8], Leather [28], [63], [64], [65].

- Shoe making [60], Fabric [66], Lace [67].

- Automated carpet/tiling cutting based on room floor plan.

- Punch press control

morphology to automated packing. The representation of the shapes to be packed as sets represents a powerful shape manipulation mechanism.

The review papers outlined indicate that there is a wide range of heuristic based approaches, but features common to the majority of these techniques include shape orientation and ordering prior to geometric manipulation of the shapes. The realization of a flexible packing system within this framework, requires the implementation of a systems engineering approach. This is necessary in order to simplify the orientation and ordering tasks.

\section{SYSTEM IMPLEMENTATION}

The packing techniques and applications discussed in the Sections II and III provide an important motivation to the development of automated packing systems. They also indicate the best means of progressing the development of flexible packing systems. The characteristics required in a flexible packing system are summarized below:

- Adaptive and easily tuned to specific applications.

- Ability to manipulate all shapes efficiently.

- Shape ordering and orientation capabilities.

- Visual feedback to aid operation.

- Ability to account for systems issues, such as changes in the product, process and the industrial environment.

- Quantitative validation procedure.

- Easy to use, interactive environment to facilitate experimentation.

- Limited propagation of errors (especially important for large packing/assembly tasks) [32].

- High speed operation.

- Facility to implement nonlocal packing strategies.

Essentially, such a system will consist of two main components. The first will provide a means of manipulating the shape and scene image at a geometric level. The second component will consist of a rule based geometric reasoning 


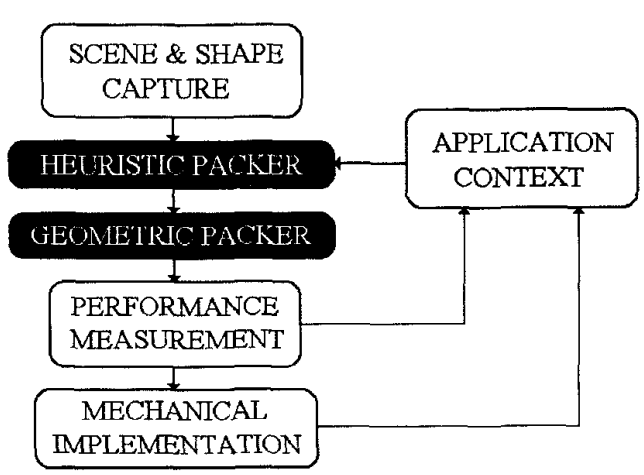

Fig. 3. General industrial packing strategy.

unit capable of deciding the ordering and orientation of the shapes to be packed. Such heuristic issues are common to many of the techniques reviewed. The heuristic component must also be capable of dealing with the system issues arising from a specific application demand. As seen from the previous discussion, this task can be simplified by maximizing the use of the information available from the product, process and the environment for a specific industrial application. By using heuristic methods it is hoped that the packing systems generality is improved and that the development of procedures for new applications will become less cumbersome. One of the key features in such a system is that it should work toward an efficient solution, accepting that we cannot guarantee reaching an optimal solution. Therefore, a mechanism for quantifying the packing systems performance will be necessary. This will enable a quantitative comparison of packing procedures. Burdea and Wolfson [32] suggest that the integration of such a heuristic approach with a packing verification procedure should ensure convergence to an efficient solution.

It is within the context of the preceding review, that an industrial packing strategy has been developed. The packing scheme consists of two major components (see Fig. 3):

1) A geometric packer, based on the principles of mathematical morphology, takes an arbitrary shape in a given orientation and puts the shape into place in that orientation [68], [69].

2) A heuristic packer which is concerned with the ordering and alignment of shapes prior to their application to the geometric packer. This component also deals with other general considerations, such as the conflict in problem constraints and the measurement of packing performance. In addition, it deals with practical constraints, such as the effects of the robot gripper on the packing strategy, packing in the presence of defective regions, and pattern matching considerations [70]. A more detailed discussion of the system implementation can be found in [65] and Whelan and Batchelor [71].

Together, these form a flexible strategy that allows the packing of arbitrary, two dimensional shapes in a previously undefined scene. The aim of the design was to produce a flexible system capable of dealing with the majority of industrial packing problems, and as such, the system was not designed around a specific application. As Burdea and Wolfson [32] point out, no single strategy, however efficient, will succeed in dealing with all shapes equally well. Therefore, when faced with a specific application, the system can be tuned to that task.

\section{A. Geometric Packer Implementation}

The concept of enclosing an arbitrary shape within its bounding rectangle, convex hull or polygon approximation (known as paver polygons) is common to many of the irregular packing techniques examined in Sections II and III. Since these techniques involve the packing of the paver polygons rather than the original shape, only approximate packing solutions can be generated due to the loss in original shape information. Other strategies, such as contour matching, describe the shapes in terms of their critical points or chain codes. Although such approaches tend to be precise, they are also computationally expensive, especially for complex shapes. For this reason, these techniques are rarely implemented on complex shapes without some degree of shape approximation.

It would be advantageous to avoid using such estimates of the arbitrary shape. Therefore an approach that deals directly with shapes would be of great benefit in the development of a flexible packing strategy. Such an approach can be found in the set-theoretic concepts of mathematical morphology [72], [73] which is concerned with the extraction or imposition of shape structure. One of the key features of the application of morphological operations to automated packing, is that the shape to be packed and its scene, do not have to be formally described to enable their manipulation by the packing system.

The function of the geometric packer is to take any arbitrary shape in a given orientation, and to put that shape into place in the scene, efficiently in that orientation. Providing the shape(s) to be packed, and the scene to be examined can be captured and stored as binary images, then morphological techniques can be applied to these images. These techniques allow the packing of a structuring element into a given image scene. In the case of the automated packing system, the shape to be packed will be represented by a morphological structuring element, while the scene will be represented by an image set on which this structuring element will act.

In the sample geometric packing problem illustrated in Figs. 4 and 5, the image scene is a rectangular bounded region (in which a star shape has already been packed), and is denoted by the image set $A$. The star shape to be packed, is applied to the image set $A$, and is denoted by the structuring element $B$. The image scene $A$ is eroded by the structuring element $B$, to produce the erosion residue image $C$. Every white pixel in this residue represents a valid packing location, Fig. 4 . The erosion residue image is then scanned, in a raster fashion, for the location of the first (white) pixel. This location is denoted by (fitx, fity). Experimentation has shown that further erosion of the residue image $C$ by a standard $3 \times 3$ square structuring, prior to searching for the first packing location, enables control of the spacing between the packed shapes. That is, the number of pixel stripping operations, on the erosion residue, is related to the spacing between the packed shapes. This relationship can also be shown mathematically [72]. The translation of the shape to be packed, $B$, to the location (fitx, fity) effectively places $B$ at the coordinate of the first possible packing location 


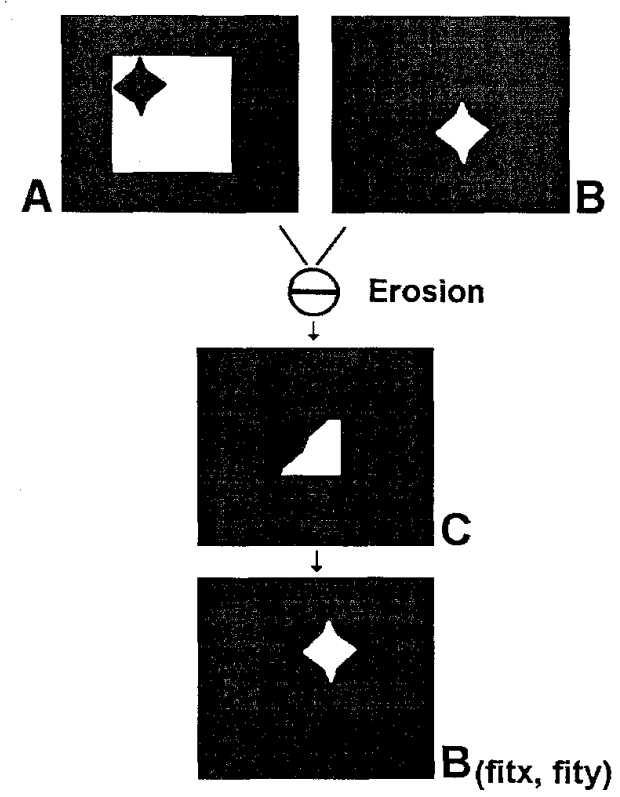

Fig. 4. Intermediate steps involved in packing a star shape in a rectangular scene. Image $A$ is eroded by shape $B$ to produce the erosion residue $C$. Shape $B$ is then relocated to (fitx, fity).

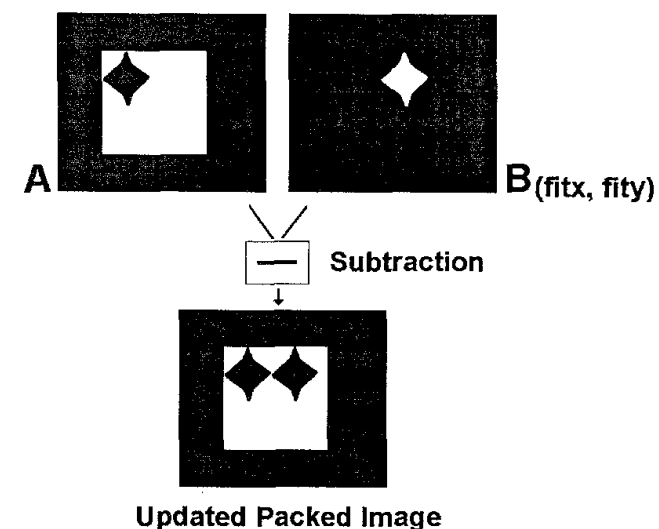

Fig. 5. Combination of the translated shape $B_{(f t x, \text { fity) }}$ with the previous scene to generate the updated packed image.

of $B$ in the scene $A$. This image is denoted by $B_{(f i t x, f i t y)}$. The resultant image is subtracted from the original image set $A$ to produce a new value for the image set $A$, therefore, effectively packing $B$ into the scene, see Fig. 5. This procedure can then be reapplied to the image set $A$ until an attempt to pack all the input shapes has been made.

\section{B. Heuristic Packer Implementation}

As outlined earlier, the heuristic packer, is concerned with the ordering and alignment of shapes prior to the application of the geometric packer. The heuristic packer operates on two classes of shapes: blobs (shapes with a high degree of curvature and/or significant concavities) and simple polygons. Details of these procedures can be found in [71]. It is necessary to consider both these general shape classes separately, since no single scheme exists for all shapes, and while the geometric packer is independent of the shape class and application context, the heuristic packer is not. The heuristic component

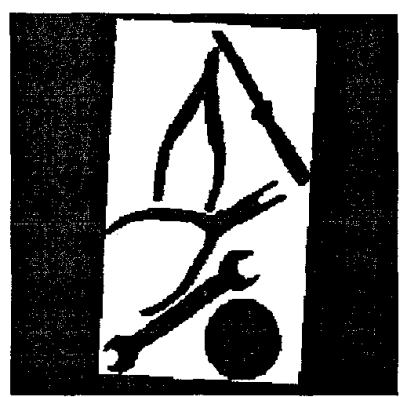

(a)

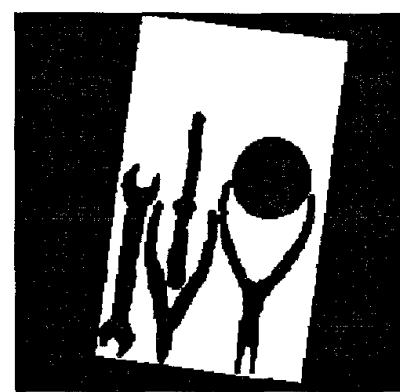

(b)

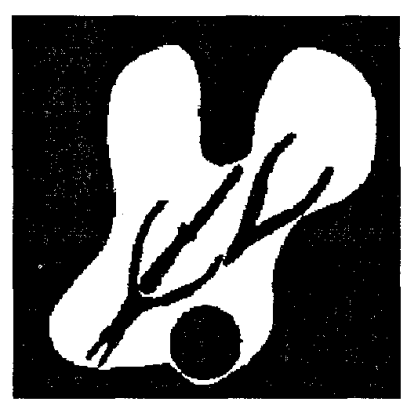

(c)

Fig. 6. Packing of tool shapes: (a) Packed in their current orientation, (b) reoriented using heurstic packing, and (c) tools packed in an irregular scene.

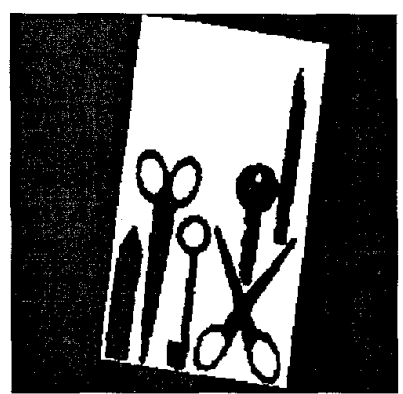

(a)

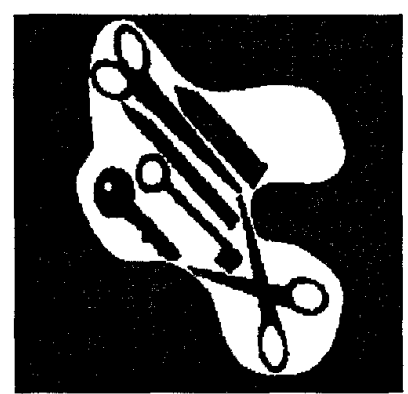

(b)
Fig. 7. Packing items into (a) a regular tray, and (b) an irregular scene.

also deals with other general considerations, such as the conflict in problem constraints, and the measurement of packing performance. In addition, it deals with a number of practical issues, such as the effects of the robot gripper on the packing strategy [69], packing in the presence of defective regions [70], [71], anisotropy and pattern matching considerations.

Fig. 6(a) shows the result of packing hand tools into a rectangular tray. These shapes were initially presented directly to the geometric packer, without the aid of the heuristic packer. This has the effect of packing each tool at whatever orientation it was in when it was presented to the vision system. Fig. 6(b) shows the resultant packing configuration when the heuristic packer precedes the geometric packer; each shape is aligned and ordered, before it is applied to the geometric packer. Fig. 6(c) shows the packing of the tools into a "random" blob region. The full packing strategy was used again here, as in Fig. 6(b). Fig. 7 shows the results of packing some standard household items, such as scissors, keys and pens, into a rectangular tray and an irregular scene. 


\section{PERformanCE MEASUReS}

To ensure that we have confidence in the global efficiency of any packing strategy, there must be some way of measuring its performance. Traditionally, packing performance has been measured by a single number, called the packing density [74]. This is the ratio of the total area of all the packed shapes to that of the total area of the scene. This is referred to as the worst case analysis packing measure.

A number of other performance measures have been developed in the field of operational research, particularly for comparing different heuristics for packing rectangular bins by odd sized boxes [2]. These performance metrics fall into two main categories: Probabilistic and statistical analysis [75]. While these performance measurements can be quite useful in well constrained packing problems, they are of little use in dealing with the packing of arbitrary shapes. Since it is unlikely that real data will fall neatly into a uniform, or any other easily analyzable distribution.

The performance measures used in our strategy are based on the traditional worst case analysis. After a packing procedure has been applied to a given scene, the result is assessed by a number of performance parameters [71].

- Packing density is the ratio of the total area of all the shapes packed, to the area of their (collective) convex hull after packing (minus the area of the scene defects). This measure has a maximum value of 1 .

- The performance index is a modified version of the packing density. A weighting factor is applied. This is referred to as the count ratio and is defined as the ratio of the total number of shapes packed, to the number of shapes initially presented to the scene. The performance index is equal to the product of the packing density, and the count ratio. The performance index also has a maximum value of 1 . This measure accounts for any shapes that remain unpacked when the procedure terminates.

\section{SYSTEMS ISSUES}

In general, the design of the packing system can be greatly simplified, the more application constraints that can be incorporated into the heuristic packer. The design of packing systems for certain industries, such as shoe manufacturing, is made easier by the fact that leather, like fabric, wood, marble and many other natural materials has a pronounced grain. For example, in certain applications only two orientations of a given shoe component may be permissible, a fact which can greatly enhance the speed of the packing procedure. Again, the heuristic packer can easily take this type of application constraint into account. Alternatively, some practical considerations can increase the complexity of the packing procedure. This section will examine two such considerations:

- The effect of utilizing different robotic end-effectors in automated packing.

- Dealing with defective scenes.

\section{A. Robot Gripper Considerations}

A general purpose packing strategy must be robust enough to cope with a range of different material handling systems.
For all of the applications considered above, it has been tacitly assumed that some form of suction or magnetic gripper could be used to lift and place the objects during packing. In this case, the foot-print of the gripper is assumed to lie within the outer edge of the shapes being manipulated.

Automated material handling systems frequently make use of robotic grippers which have two or more fingers. This complicates the problem of packing, since the gripper requires access to objects within a partially packed scene. Therefore, any packing strategy must make allowances for the gripper. The grippers worse case position usually (but not always) occurs when the gripper is fully open, just after placing an object in position. The problem of gripper access can be dealt with very effectively, by the simple expedient of overlaying a gripper template on the shape to be packed prior to the application of the geometric packer. The gripper foot-print is based on the positions of the fingers in both the open and closed positions. In fact, the convex hull of each of the finger tips in the open and closed positions is formed when computing the composite foot-print [71].

In a practical situation, care must be taken to ensure that any change in the shape of the objects to be packed, due to squeezing by the robot gripper, does not adversely affect the packing strategy. The same is true of articulated and other hinged objects, such as scissors or pliers, which can change their shape during handling. Again, this type of application constraint could also be dealt with by the introduction of suitable heuristic packing rules, and may also be used as a factor when calculating the gripping position.

The strategy outlined above for working with multi-finger grippers does have the advantage of allowing the shapes to be unpacked from the scene in any order. One possible modification to this approach results in a denser configuration that, in general, can only be automatically unpacked in reverse order. This modification consists of packing each shape, taking the robot foot-print into account, but removing the foot-print from the scene prior to the application of the next shape.

Of course, many industrial applications do not require that the pieces are unpacked automatically. Certain industrial tasks require manual unpacking, and consideration of the means and the environment of the manual unpacking operation may influence the automatic packing strategies used. For example, if the application requires that items are to be unpacked in a certain order, then this will influence the packing strategy.

Consideration must also be given to the means in which the shapes are initially acquired by the robot. If the objects are presented one at a time, then the system can automatically determine the optimal gripper pickup point (robot grasping location) for that object, and proceed to pack it using one of the automated packing strategies outlined previously.

Alternatively, if all the objects to be packed are placed in the field of view at the same time then it may not always be possible for the multi-fingered robot to access the piece it is required to pack. The vision system may have to guide the robot gripper to push the object of interest until it is separated enough from the other shapes for the gripper to gain access to the desired pickup points. Also the order in which the shapes are packed may be determined by the order in which the robot 


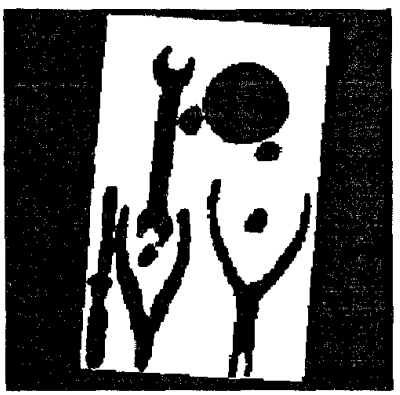

(a)

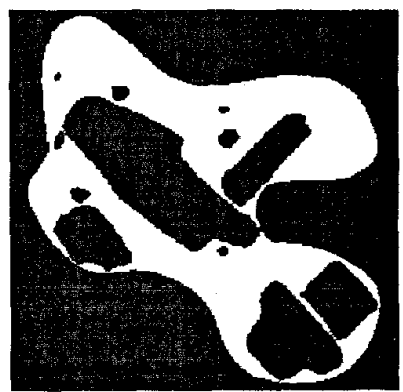

(b)
Fig. 8. Packing items into defective regions: (a) Tools in a defective rectangular tray, and (b) cutting jacket template pieces from an irregular fabric segment which contains defective regions

can access the objects. Once the shapes have been packed, the robot end-effector can also be used to nudge the packed shapes such that a more efficient packing configuration can be achieved.

\section{B. Packing Scenes with Defective Regions}

Any practical automated packing system for use in such industries as leather or timber processing must be able to pack objects into a scene which may contain defective regions. The heuristic packer can readily accommodate defective regions; by simply defining the initial scene to contain a number of holes. Fig. 8(a) illustrates the effect of packing tools into a rectangular tray which contains four small blob-like defects. By comparing this to the packing configuration shown in Fig. 6(b), it is clear that the packing is not as compact when defects are taken into account (as you would expect).

Fig. 8(b) shows the packing of jacket template pieces on to a piece of fabric, prior to cutting. The small blob-like regions indicate the defective areas in the fabric. These defective regions are not to be included in the jacket pieces to be cut. These results illustrate the flexibility of the packing strategies outlined in Section IV.

\section{DISCUSSION}

The basic concepts discussed in this paper attempt to lay a solid foundation for further research in the area of automated packing, and the development of intelligent machine vision systems. Further directions of research include:

\section{A. Enhancements to the Current Packing Strategy}

The next logical step in the development of the packing strategies outlined, concerns improvement of the basic packing components. With regard to the geometric packer, there is the need to investigate the effect of alternative erosion residue scanning techniques. Likewise, with the heuristic packer, the investigation of more complex heuristics may lead to further improvements, although one must be careful not to overburden the system with overly complex procedures that would reduce the computational efficiency of the system. Whenever possible full use should be made of the systems engineering approach to avoid unnecessary complexity. The functionality, and efficiency, of the heuristic packer may be further improved with the incorporation of some more of the ideas of computational geometry, and the development of new packing strategies based on the lessons learnt from this work.

Once the packing strategy has been implemented for a given application, it may be possible to undergo a shakedown stage that will enable a further increase in packing efficiency. While this may not always be physically possible, for example due to the nature of the handling equipment or the fragile nature of the objects being packed, software implementations of this shakedown stage may present a means to further increase the performance of the packing procedures.

The extension of the packing strategy to deal with grey scale images also presents a significant challenge. Again, the key to this enhancement lies in the integration of grey scale image analysis techniques, such as grey scale morphology, to the geometric packer. The implementation of the grey scale equivalents of the binary morphological packing techniques may simplify some of the decision making in the heuristic packer.

\section{B. The Three-Dimensional Packing Problem}

The investigation discussed in this paper, has concentrated on the packing of two dimensional shapes, and an obvious extension of this work is the application of these ideas to the three-dimensional packing problem. The development of a three-dimensional packing strategy will require a number of changes to the existing packing procedures. An investigation into the systems issues involved in the implementation of a three-dimensional packing system, such as the use of structured lighting to gain the three-dimensional representation and the increase in complexity of the material handling operations, will also be necessary.

One of the obvious techniques when moving from two to three-dimensional packing problems, involves the use of the two dimensional procedures on a layered basis. While this may produce some useful results, a better insight into the difficulties of the task may be developed by tackling the problem from first principles. Many of the practical systems issues that are outlined in this paper will still apply.

\section{Packing and Automated Assembly}

There has been a general trend, when applying automation to a range of industries, to aim for full automation of the process. In reality this is not always likely to be achieved, primarily because of the difficulty in accommodating assembly tasks. Since many assembly tasks are too complex to automate completely using today's technology, it is much more realistic to consider assembly to be a task that requires people and machines working in unison. Therefore, semiautomated assembly systems have an important role to play in the development of flexible manufacturing systems. The heuristic approach is well suited to such semi-automated systems. The automated packing system can be easily modified to accommodate such an approach to the assembly task. For example, some of the more complex geometric reasoning (currently implemented in the heuristic packer) may be better performed by the human operator, whereas the geometric packer is better able to control the inter-object or robot gripper spacing. 


\section{Packing for Aesthetic Appeal}

Further extensions of this work include the development of systems that will allow packing not only for efficiency, but for aesthetic appeal [71]. Investigation into this research area involves the development of rule based systems that can measure the attractiveness of packaging, or a given packing configuration. For example, the automated laying of a wooden floor and the automated packaging of food. A key challenge in this research is to develop a means of dealing with a subjective criteria such as attractiveness in an automated way.

\section{E. Packing Non-Planar Objects}

One key concern involves the packing of nonplanar objects. That is, an object may not lie flat in the plane, therefore requiring a perspective transformation prior to the application of a geometric packing process. For example, overlapping objects, or objects resting at an angle against the side of a box. The combination of the packing strategy outlined in this paper, along with the growing body of research work on shape invariance may provide a useful strategy for tackling such perspective problems. An alternative approach may consist of the robotic manipulation of the object so that it lies in the plane prior to the application of the packing process.

\section{CONCLUSION}

The work outlined in this paper was motivated by the need to produce flexible industrial vision systems. The application chosen to act as a test bed domain for these ideas was the automated packing of irregular shapes. The prototype system had to be capable of implementing efficient packing strategies, with no prior knowledge of the shapes to be packed or the scenes into which the shapes were to be placed. Such a system needs to be flexible, and must have the ability to deal with a wide range of possible shapes, therefore requiring powerful shape handling mechanisms.

The approach outlined in this work is unique in that it combines the strengths of the heuristic approaches to problem solving, with a powerful shape manipulation mechanism, namely mathematical morphology. While it is acknowledged that the application of mathematical morphology to the packing of a small number of arbitrary planar shapes is not new, researchers in this area have concentrated on the mathematical proof of optimal packing configurations, rather than viewing it within the context of the industrial packing problem. But since a mathematical proof that is capable of dealing with a large number of arbitrary shapes may not exist, the application of a heuristic approach that allows the targeting of an efficient rather than an optimal solution, may be a more fruitful line of research.

The second major theme running through this work is the benefits that can be gained by applying a systems engineering approach to complex vision applications. As has been found so often in other applications, the adoption of such an approach is an invaluable aid in the design of industrial vision systems. A systems approach to the design of the packing strategy has been deliberately taken. By taking the systems constraints into account, faster, cheaper solutions may be obtained. It is our view that automated packing systems are best designed with regard to such issues as gripper shape, material grain, material defects, etc. It is believed that there is little justification for seeking a unified algorithmic solution that is capable of tackling the totality of packing applications, without human intervention.

\section{REFERENCES}

[1] H. L. Dreyfus and S. E. Dreyfus, Mind over Machine. New York: Free Press, 1986

[2] W. B. Dowsland, "Two and three dimensional packing problems and solution methods," New Zealand Opl. Res., vol. 13, no. 1, pp. 1-18, 1985.

[3] Adept, ADEPT Case Studies no. 101-108, ADEPT Tech. Inc., 1991.

[4] P. E. Sweeney and E. R. Paternoster, "Cutting and packing problems: A categorized, application-orientated Res. bibliography," J. Opl. Res. Soc. vol. 43 , no. 7, pp. 691-706, 1992.

[5] K. A. Dowsland and W. B. Dowsland, "Packing problerns," Europ. J. Opl. Res., vol. 56, pp. 2-14, 1992.

[6] E. A. Silver, R. V. V. Vidal, and D. de Werra, "A tutorial on heuristic methods," Europ. J. Opl. Res., vol. 5, pp. 153-162, 1980.

[7] H. Dyckhoff, "A typology of cutting and packing problems," Europ. J. Opl. Res., vol. 44, pp. 145-159, 1990.

[8] A. R. Brown, Optimum Packing and Depletion: The Computer in Space-and Resource-Usage Problems. Amsterdam: Elsevier, 1971.

[9] E. L. Hinrichsen, J. Feder, and T. Jossang, "Random packing of disks in two dimensions," Phys. Rev. A, vol. 41, no. 8, pp. 4199-4209, 1990.

[10] H. L. Ong, M. J. Magazine, and T. S. Wee, "Probabilistic analysis of bin packing Heuristics," Opl. Res., vol. 32, no. 5, pp. 983-998, 1984.

[11] H. Carpenter and W. B. Dowsland, "Practical considerations of the pallet-loading problem," J. Opl. Res. Soc., vol. 36, no. 6, pp. 489-497, 1985.

[12] C. S. Chen, S. Sarin, and B. Ram, "The pallet packing problem for nonuniform box sizes," Int. J. Prod. Res., vol. 29, no. 10, pp. 1963-1968, 1991.

[13] F. R. K. Chuang, M. R. Garey, and D. S. Johnson, "On packing two dimensional bins," SIAM J. Alg. Disc. Meth., vol. 3, no. 1, pp. 66-76, 1982

[14] B. S. Baker, E. G. Coffman, and R. L. Rivest, "Orthogonal packing in two dimensions," SIAM J. Comput., vol. 9, no. 4, pp. 846-855, 1980.

[15] E. E. Bischoff and M. D. Marriott, "A comparative evaluation of heuristics for container loading," Europ. J. Opl. Res., vol. 44, pp. 267-276, 1990.

[16] E. Hall, R. Shell, and G. Slutzky, "Intelligent packing and material handling," Proc. SPIE Intelligent Robots and Computer Vision IX: Algorithms and Techniques, vol, 1381, pp. 162-170, 1990.

[17] R. C. Wilson, "A packaging problem," Mgmt. Sci., vol. 12, no. 4, pp B135-B145, 1965.

[18] E. E. Bischoff, "Interactive approaches to packing problems," I0th ICPR, pp. 55-61, 1989.

[19] B. G. Batchelor, Intelligent Image Processing in Prolog. London: Springer-Verlag, 1991.

[20] W. Qu and J. L. Sanders, "A nesting algorithm for irregular parts and factors affecting trim losses," Int. J. Prod. Res., vol. 25, no. 3, pp. 381-397, 1987.

[21] D. Dori and M. Ben-Bassat, "Efficient nesting of congruent convex figures," Commun. ACM, vol. 27, no. 3, pp. 228-235, 1984

[22] B. Chazelle, "The polygon containment problem," Adv. Comput. Res. vol. 1 , pp. 1-33, 1983

[23] F. Koroupi and M. Loftus, "Accommodating diverse shapes within hexagonal pavers," Int. J. Prod. Res., vol. 29, no. 8, pp. 1507-1519, 1991.

[24] R. R. Martin and P. C. Stephenson, "Putting objects into boxes," CAD. vol. 20, no. 9, pp. 506-514, 1988 .

[25] Y. K. D. V. Prasad and S. Somasundaram, "CASNS-A heuristic algorithm for the nesting of irregular shaped sheet-metal blanks," Comp. Aid. Eng. J., vol. 8, no. 2, pp. 69-73, 1991.

[26] W. W. Chow, "The packing of a template on a flat surface," Trans. ASME J. Mechan. Desn., vol. 102, pp. 490-496, 1980.

[27] R. Kothari and P. Klinkhachorn, "Packing of convex polygons in a rectangularly bounded, nonhomogeneous space," IEEE Proc. 21 st Southeastern Symp. on System Theory, pp. 200-203, 1989.

[28] A. Albano and G. Sapuppo, "Optimal allocation of two dimensional irregular shapes using heuristic search methods," IEEE Trans. Syst. Man, Cybern., vol. 10, no. 5, pp. 242-248, 1980.

[29] L. Vincent, "Morphological transformations of binary images with arbitrary structuring elements," Signal Processing, vol. 22, pp. 3-23, 1991 
[30] C. Malcolm and T. Smithers, "Symbol grounding via a hybrid architecture in an autonomous assembly system," Robot. Auton. Syst., vol. 6, pp. $123-144,1990$.

[31] S. R. Oh, J. H. Lee, K. J. Kim, and Z. Bien, "An intelligent robot system with jigsaw-puzzle matching capability," in Proc. 15th Int. Symp. on Indust. Robots, Tokyo, 1985, pp. 103-112.

[32] G. C. Burdea and H. J. Wolfson, "Solving jigsaw puzzles by a robot," IEEE Trans. Robot. Automat., vol. 5, no. 6, pp. 752-764, 1989.

[33] H. Wolfson, E. Schonberg, A. Kalvin, and Y. Lamdan, "Solving jigsaw puzzles by computer," Annals Oper. Res., vol. 12, pp. 51-64, 1988.

[34] N. R. Hudson, C. F. Kelly, E. McQuade, and M. A. Rahman, "Application of artificial intelligence to two dimensional assembly tasks," in Al Cogn. Sci. '89, 1989, pp. 3-19.

[35] M. H. Lee, Intelligent Robotics, Open Univ. Press Robotics Series, 1989.

[36] F. J. M. Van der Heijden, "Assembly of small components by a visioncontrolled robot using the Philips vision system PAPS," in Proc. 5th Int. Conf. Robot Vision and Sensory Controls, 1985, pp. 517-528.

[37] R. Shoureshi, M. Momot, O. R. Mitchell, and J. Feddema, "Vision-based intelligent control for automated assembly," J. Intell. Robot. Syst., vol. 2 , pp. $153-170,1989$

[38] A. Delgrange and S. Maouche, "Fast vision algorithm for shirt col lar automatic assembling cell," in Proc. IASTED Intl. Symp. Applied Informatics-Al' 89,1989 , pp. 93-96.

[39] E. L. Hall, G. D. Slutzky, and R. L. Shell, "Intelligent robots for automated packaging and processing," in Proc. ASME 1989 Pressure Vessels and Piping Conf., 1989, pp. 141-146.

[40] A. Jain and M. Donath, "Knowledge representation system for robotbased automated assembly," J. Dynamic Syst., Measure. Contr., vol. 111 , pp. $462-469,1989$.

[41] R. Hoffman, "Automated assembly in a CSG domain," in IEEE Intl. Conf. on Robot. Automat., 1989, vol. 1, pp. 210-215.

[42] H. Hitakawa, "Advanced parts orientation system has wide application," Assembly Automat, vol. 8, no. 3, pp. 147-150, 1988.

[43] C. L. P. Chen, "Automatic assembly sequences generation by pattern matching," IEEE Trans. Syst., Man. Cybern., vol. 21, no. 2, pp. 376-389, 1991.

[44] A. C. Kak, K. L. Boyer, C. H. Chen, R. J. Safranek, and H. S. Yang, "A knowledge-based robotic assembly cell," IEEE Expert, pp. 63-83, 1986.

[45] N. Ayache and O. D. Faugeras, "HYPER: A new approach for the recognition and positioning of two dimensional objects," IEEE Trans. Pattern Anal. Machine Intell., vol. 8, no. 1, pp. 44-54, 1986.

[46] C. G. Geschke, "A system for programming and controlling sensorbased robot manipulators," IEEE Trans. Pattern Anal. Machine Intell., vol. 5, pp. 1-7, 1983

[47] E. North Coleman, Jr. and R. E. Sampson, "Acquisition of randomly orientated workpieces through structured mating," Proc. Comp. Vis. Pattern Recog., pp. 350-357, 1985.

[48] J. L. Morris, "Vision applications of a direct drive robot," in Proc. 5th Int. Conf. Robot Vision Sensory Control, 1985, pp. 575-585.

[49] G. F. Cowan, D. M. Chelberg, and H. S. Lim, "ACRONYM model-based vision in intelligent task automation project," in Proc. IEEE Conf. on Robotics and Automation, 1984, pp. 176-183.

[50] B. Gardstam, "Car body assembly with ASEA 3D vision," in Proc. 5th Int Conf. Robot Vision and Sensory Cntrl, 1985, pp. 509-517.

[51] P. A. Fehrenbach, "Optical alignment of dual-in-line components for assembly," in Proc. 3rd Int. Conf. Robot Vision Sensory Control, 1983, pp. 609-615.

[52] K. Tamaki and Y. S. Nof, "Design method of robot kitting for flexible assembly," Robot. Auton. Syst., vol. 8, no. 4, pp. 255-273, 1991.

[53] F. Y. Zheng, R. Pei, and C. Chen, "Strategies for automatic assembly of deformable objects," in Proc. '91 Int. Conf. Robotics and Automation, 1991, vol. 3, pp. 2598-2603.

[54] D. R. Hoska, "Fixtureless assembly/manufacture," in Proc. Robots and Vision Automation' ' 90,1990 , pp. 4-17-4-28.

[55] J. D. Tedford, "Developments in robot grippers for soft fruit packing in New Zealand," Robotica, vol. 8, no. 4, pp. 279-183, 1990.

[56] I. Stewart, "How to succeed in stacking," New Scientist, pp. 29-32, July 1991.

[57] K. Khodabandehloo, "Robotic handling and packaging of poultry products," Robotica, vol. 8, no. 4, pp. 285-297, 1990.

[58] M. Kassler, "Robotics and prawn-handling," Robotica, vol, 8, no. 4, pp. 299-301, 1990.

[59] H. Deleplanque, P. Bonnet, and J. G. Postire, "An intelligent robotic system for in vitro plant production," in Proc. 5th Int. Conf. Robot Vision Sensory Control, 1985 , pp. 305-313.

[60] R. Cuninghame-Green, "Geometry, shoemaking and the Milk Tray problem," New Scientist, pp. 50-53, Aug. 12, 1989.

[61] W. B. Dowsland, "Three-dimensional packing - Solutions approache and heuristic development," Int. J. Prod. Res., vol. 29, no. 8, pp.
$1673-1685,1991$

[62] B. Carré, private communication, 1993

[63] S. Dulio, "Application of automation technology to leather cutting," in Proc. 23rd Int. Symp. Auto. Tech. Automation, 1990, pp. 83-96.

[64] J. Chung, D. Scott, and D. J. Hillman, "An intelligent nesting system on 2-D highly irregular resources," in Proc. SPIE Applica. Art. Intell. VIII, 1990, vol. 1293, pp. 472-483.

[65] P. F. Whelan, Automated Packing of Arbitrary Shapes: A Systems Engineering Approach, Ph.D. thesis, Univ. of Wales, College of Cardiff, 1994.

[66] J. Uhry, "Applications of simulated annealing in operation research," in New Methods in Optimization and their Industrial Uses. Berlin: Birkhauser-Verlag, 1989, pp. 191-203.

[67] R. A. Russell and P. Wong, "Automation of lace cutting using computer vision," in Proc. Int. Symp. Expo. Robots. 1988, pp. 385-393.

[68] P. F. Whelan and B. G. Batchelor, "Automated packing of arbitrary shapes," in Machine Vision Systems: Integra. Appl. Proc. SPIE, 1991, vol. 1615 , pp. $77-86$.

[69] _ "Development of a vision system for the flexible packing of random shapes," in Machine Vision Appl. Arch. Syst. Int. Proc. SPIE, 1992, vol. 1823, pp. 223-232.

[70] _ "Automated packing systems: Review of industrial implementations," in Machine Vision Appl. Arch. Syst. Int. II, Proc. SPIE, 1993, vol. 2064 , pp. $358-369$.

[711 _ _ "Flexible packing of arbitrary two dimensional shapes," Opt. Eng., vol. 32, no. 12, pp. 3278-3287, 1993.

[72] R. M. Haralick and L. G. Shapiro, "Mathematical Morphology," in Computer and Robot Vision: Vol. 1. Reading, MA: Addison-Wesley, 1992, ch. 5

[73] E. R. Dougherty, "An introduction to morphological image processing," SPIE Press, Tutorial Text TT9, 1992.

[74] R. Fowler, M. Paterson, and S. Tanimoto, "Optimal packing and covering in the plane are NP complete," Inf. Proc. Lett., vol. 12, no. 3. pp. $133-137,1981$

[75] M. R. Garey and D. S. Johnson, Computers and Intractability-A Guide to the Theory of NP-Completeness. San Francisco: Freeman, 1979

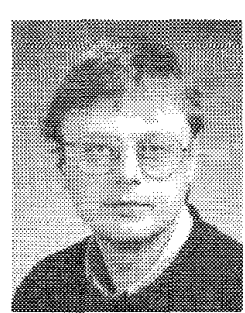

Paul F. Whelan (S'84-M'85) received the B.Eng. (Hons.) degree from the National Institute for Higher Education Dublin, Ireland, the M.Eng. degree in machine vision from the University of Limerick, Ireland, and the Ph.D. from the University of Wales, College of Cardiff, in 1985, 1990, and 1994 , respectively.

From 1985 to 1989 , he was with Industrial and Scientific Imaging Ltd, where he was involved in the research and development of industrial vision systems. He was later with Westinghouse as a senior design engineer on their range of machine vision inspection products. In August 1992, he was appointed to the Faculty of Engineering and Design, Dublin City University. Since joining DCU, he has been active in the founding and development of the Vision Systems Group. His research interests include image analysis, machine vision and systems engineering.

Dr. Whelan was the co-editor of Selected Papers on Industrial Machine Vision Systems. He is a member of SPIE, IEE, and a senior member of the SME (Machine Vision Association)

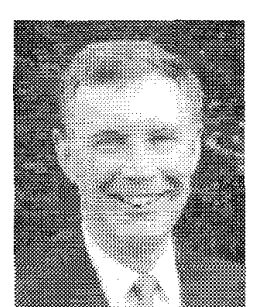

Bruce G. Batchelor received the B.Sc. and Ph.D degrees from the University of Southampton, England, in 1965 and 1969 , respectively.

He was with the Electronics Research Laboratory, Plessey Company Ltd., from 1968 to 1970 . In January 1971, he was appointed to a Lectureship in Electronics at the University of Southampton. From 1980 to 1992, he was full Professor of Electronic Engineering, University of Wales, Cardiff, and is currently Professor of Computing Mathematics, in the same institution. His research interests are in the application of intelligent image processing techniques to industrial inspection and control. He has published five books and more than 200 technical articles on automated visual inspection, machine vision systems and pattern recognition.

He is a fellow of the SPIE, SME, IEE, and BCS. 